oder die hübschen Damen, die in der «Baixa luxuriöse Schaufenster bewundern, oder die Geschäftsherren, die sich tagtäglich in den «Männercafés〉 zum Plauderstündchen einfinden.

E. Schwabe (Bern): Der Fremdenverkehr im 'Atlas der Schweiz).

Der Bedeutung des Fremdenverkehrs in unserm Land und seinem starken Aufschwung in den letzten Jahren sucht der «Atlas der Schweiz〉 mit einer doppelseitigen Karte im Maßstab 1:500 000 und einer Reihe von Nebenkarten und -plänen gerecht zu werden, die sich gegenwärtig in Ausarbeitung befinden. Dabei wird besonderes Gewicht nicht allein darauf gelegt, die vielfältigen Erscheinungen des Tourismus im Landschaftsbilde darzustellen, sondern zu zeigen, wie sehr dieser Wirtschaftszweig heute gewisse ursprünglich rein landwirtschaftlich ausgerichtete Gegenden, dazu auch kleinere und größere städtische Gemeinwesen durchdringt. Unter anderem sollen die in bestimmten Regionen und Jahreszeiten akzentuierten Bevölkerungsballungen in der Karte zum Ausdruck kommen, die ganz eigenen Problemen gerufen haben; auch soll bei den wichtigern touristisch orientierten Orten wenn möglich die Aufgliederung des Beherbergungspotentials nach Hotels, Privatunterkünften, Instituten und so weiter dargelegt werden.

W. Kündig-Steiner (Zürich) : Die Oelpalmen-Versuchsstation des SRMO bei Douala (Kamerun). Paraîtra dans Geographica Helvetica, voir ce numéro page 202.

E. Bienz (Basel) : Der Strukturatlas Nordwestschweiz-Oberelsaß-Südschwarzwald.

Dieses im Auftrag der Kantone Basel-Stadt und Basel-Landschaft herausgegebene und am Geographischen Institut der Universität Basel bearbeitete Werk kommt auf Weihnachten 1966 zur Auslieferung. Die Mehrzahl der ca. 80 großenteils mehrfarbigen Karten umfaßt im Maßstab 1:250 000 einen Umkreis von 30-50 km um die Dreiländerecke nördlich Basel. Einzelne Karten im Maßstab 1: 500000 bedecken das wesentlich größere Gebiet der 〈Regio〉. Zum erstenmal erscheint also für diesen Raum ein Atlas, dessen thematische Karten auf Gemeindebasis alle Landesgrenzen überschreiten und somit bisher noch nirgends dargestellte Beziehungen aufzeigen. Der Inhalt der Karten reicht von naturräumlichen Themen über Bevölkerung, Siedlung, Wirtschaft und Verkehr bis zur Organisation der Orts- und Regionalplanung. Der Preis des Werkes beträgt ca. Fr. 100.-.

\title{
DIE STELLUNG DER GEOGRAPHIE INNERHALB DER ALTEN UND DER IM ENTWURF VORLIEGENDEN NEUEN MATURITÄTSVERORDNUNG
}

\author{
HANS BERNHARD
}

\section{Das alte Maturitätsreglement}

Schulreglemente sind in der Regel außerordentlich zäh und langlebig. Das gilt auch für das bestehende Eidgenössische Maturitätsreglement, das (ohne die verschiedenen Revisionen) aus dem Jahre 1925 stammt. Es ist eine Verordnung über die Anerkennung von Maturitätsausweisen für das Medizinstudium durch den Schweizerischen Bundesrat und regelt Alter, Ausbildung und Prüfung des Maturanden. Es unterscheidet die 
Typen A, B und C. Neben der Pflege der Muttersprache und einer zweiten Landessprache befaßt sich Typus A vor allem mit Lateinisch und Griechisch, Typus B mit Lateinisch und modernen Sprachen und Typus C mit Mathematik und Naturwissenschaften. Inhaber eines Maturitätsausweises nach Typus C können, sofern sie Schweizer Bürger sind, die Zulassung zu den Eidgenössischen Medizinalprüfungen durch eine Ergänzungsprüfung in Latein erwerben. Das war ein Punkt, der schon früh heftiger Kritik rief und immer wieder von sich reden machte, da man diese Verfügung vielfach eher als Berufsschutz der Mediziner denn als Notwendigkeit erachtete.

Im Maturitätsreglement von 1925 nimmt die Geographie eine Sonderstellung ein. Sie gehört mit Geschichte, Biologie, Physik und Chemie zu denjenigen Fächern, in denen die Maturitätsnote nicht unbedingt durch eine Prüfung belegt sein muß. Für die Reifeerklärung zählt aber die Geographienote (Erfahrungs- oder Erfahrungs- und Prüfungsnote) sowohl bei der Bewertung ungenügender Leistungen wie auch als Teil der erforderlichen Notensumme.

Unterschiede ergeben sich jedoch hinsichtlich des Unterrichtes auf der Oberstufe. Während dieser in den obligatorischen Prüfungsfächern und in Geschichte bis zum Ende der gesamten Schulzeit zu erteilen ist, darf der Fachunterricht in Biologie, Physik* und Chemie nicht früher als zwei Jahre, in Geographie nicht früher als ein Jahr vor der Matur abgeschlossen werden. Diese Besserstellung verdankt die Geographie in erster Linie den Bemühungen des damaligen Stadtpräsidenten von Bern, Dr. E. Bärtschi, der vorher am dortigen Gymnasium Geographie unterrichtet hatte, und um die Sorgen und Nöte dieses Faches wußte.

Diese Bevorzugung blieb mehr oder weniger unangetastet, bis ein Rektor der Kantonsschule Winterthur Übungen in kleineren Gruppen auf der Oberstufe nur unter der Voraussetzung als durchführbar erklärte, daß der Unterricht in Geographie bereits eineinhalb Jahre vor der Maturität abgeschlossen werden dürfe. Das wurde von der Eidgenössischen Maturitätskommission denn auch versuchsweise gestattet. Von diesem Fall erfuhr die Öffentlichkeit erst, als Ständerat Vaterlaus in einem Postulat, das im Dezember 1958 im Ständerat eingereicht und im April 1959 im Rate begründet wurde, unter anderem bemerkte, das Departement des Innern habe der definitiven Durchführung der Übungen zugestimmt, verlange aber, daß der Geographieunterricht wieder bis ein Jahr vor der Matur erteilt werde. Diese Bestimmung der Maturitätsverordnung erscheine wenig sinnvoll, wenn man bedenke, daß der Unterricht in Physik, Chemie und Biologie zwei Jahre vor dem Ende der Schulzeit abgeschlossen werden dürfe. Der damalige Departementschef, Bundesrat Etter, nahm das Postulat, das sich vor allem mit der Gleichberechtigung des Typus $\mathrm{C}$ befaßte, zur Prüfung entgegen.

Kurze Zeit darauf trafen sich der Verfasser (als bereits designierter neuer Präsident des Vereins Schweizerischer Geographielehrer) und K. Bösiger (der damalige Präsident) in Olten zur Abfassung einer Eingabe an das Departement des Innern. In dieser wurde der Bundesrat ersucht, die bisherige Regelung zu belassen, da die Aufgaben des Geographieunterrichtes sehr stark angewachsen seien und dieser nicht von der Oberstufe verdrängt werden dürfe. Zudem werde der Unterricht in Physik, Chemie und Biologie in den meisten Mittelschulen der Schweiz bedeutend weiter hinaufgeführt, als das nach dem Postulat Vaterlaus angenommen werden könne. - Schon fünf Tage später, am 16. Mai 1959, folgte eine Antwort von Bundesrat Etter, worin dieser wörtlich ausführte: ¿Für unser Departement besteht über die Bedeutung des Geographieunterrichtes in der Gymnasialausbildung kein Zweifel. Diese Erkenntnis werden wir mit der Eidgenössischen Maturitätskommission auch in der Behandlung des Postulates Vaterlaus nicht außer acht lassen.) Und an anderer Stelle: «Diese Tolerierung einer Abweichung von der eidgenössischen Vorschrift (gemeint ist der Versuch an der Kantonsschule Winterthur. Der Verfasser) ist jedoch wie erwähnt provisorischer Natur.

\footnotetext{
* Für Typus C ist Physik obligatorisches Prüfungsfach
} 
Nach Ablauf dieser Versuchsdauer wird darüber neu zu befinden sein.> Daraus ergab sich, daß im Gegensatz zu der im Postulat Vaterlaus erwähnten definitiven Regelung die Versuchsdauer noch nicht abgeschlossen war.

Dann hörte man in dieser Angelegenheit lange nichts mehr, bis Prof. Wy $\beta$, der Präsident der Eidgenössischen Maturitätskommission, am 27. April 1961 (genau zwei Jahre nach der Begründung des Postulates) in einem Referat vor der Konferenz der kantonalen Erziehungsdirektoren im Beisein des Vorstehers des Departementes des Innern, jetzt Bundesrat Tschudi, unter anderem ausführte: «... daß ich persönlich mit Herrn Ständerat Vaterlaus der Ansicht bin, die Bevorzugung der Geographie in bezug auf den Zeitpunkt des Unterrichtsabschlusses gegenüber Physik, Chemie und Naturgeschichte sei, wenn sie es überhaupt je war, heute nicht mehr gerechtfertigt. Aber die prompte und heftige Reaktion des Vereins Schweizerischer Geographielehrer auf die Mißachtung der einschlägigen Vorschrift der Anerkennungsverordnung am Gymnasium Winterthur ist ein Musterfall dessen, was eintritt, wenn einzelne Schulen oder Schulbehörden sich einfach über die eidgenössischen Vorschriften hinwegsetzen.>

Man kann mit Recht fragen, warum sich die Geographielehrer so sehr auf diese Besserstellung ihres Faches versteiften und sich nicht mit den Fächern Physik, Chemie und Biologie gleichzustellen trachteten. Aber man darf sich durch das Reglement nicht über die realen Verhältnisse hinweg täuschen lassen. Es besagt ja nur, wann der Unterricht in den betreffenden Fächern frühestens aufhören dürfe. Nach oben wird gar nichts vorgeschrieben. Die scheinbare und zum Teil auch ursprüngliche Schlechterstellung der drei Fächer Physik, Chemie und Biologie hat sich aber im Laufe der Jahre immer mehr in eine deutliche Bevorzugung gewandelt. Eine Umfrage, die sich 1961 auf 59 Mittelschulen der Schweiz erstreckte, ergab für die beiden letzten Schuljahre folgendes Mittel an Wochenstunden

$\left.\begin{array}{llll}\text { Geographie } & 1,78 & \text { Chemie } & 3,84 \\ \text { Biologie } & 2,37 & \text { Physik } & 4,60\end{array}\right\} \begin{aligned} & \text { inklusive Labor und } \\ & \text { obligatorische Übungen }\end{aligned}$

Auch in den Naturwissenschaften ist das Stoffgebiet gewaltig angestiegen. Zudem besitzen sie, wie Mathematik und Sprachen, starke Fürsprecher und Helfer in Wirtschaft und einflußreichen Verbänden. Für die Geographie trifft dies leider nicht zu, und deshalb ist sie mehr denn je auf eine Festigung ihrer Stellung im Maturitätsreglement angewiesen.

Diese Tatsachen bewogen Prof. Dr. H. Gutersohn und den Verfasser, Herrn Ständerat Dr. E. Vaterlaus über die ganze Sachlage aufzuklären und ihm darzulegen, daß die beanstandete Besserstellung der Geographie schon längst nur noch eine scheinbare sei. Diese Besprechung fand am 29. November 1962 in Zürich statt. Ständerat Vaterlaus schien unseren Standpunkt zu begreifen und war unserer Sache nicht abgeneigt. Er gab uns sogar einen Hinweis, wo und wann wir weiter Stellung beziehen sollten. Leider schien er das in seinem Artikel vom 3. Juli 1964 in der «Neuen Zürcher Zeitung» wieder vergessen zu haben.

\section{Revision der Eidgenössischen Verordnung über die Anerkennung der Maturitätsausweise}

Am 9. September 1963 ernannte das Eidgenössische Departement des Innern eine Expertenkommission für die Revision der Maturitäts-Anerkennungsverordnung. Sie setzte sich aus Mitgliedern der Erziehungsdirektorenkonferenz, der Eidgenössischen Maturitätskommission, der Hochschulrektorenkonferenz, der Gymnasialrektorenkonferenz, des Vereins Schweizerischer Gymnasiallehrer und der Verbindung der Schweizer Ärzte zusammen und wurde von Prof. Dr. Karl Schmid von der Eidgenössischen Technischen Hochschule präsidiert. Diese Kommission gliederte sich für einen Teil 
ihrer Arbeit in drei Subkommissionen, die sich nun in größtes Schweigen hüllten. Wenn es im Bericht Schmid heißt «Im übrigen hatten die Subkommissionen die ausdrückliche Kompetenz, mit Persönlichkeiten, die nicht der Expertenkommission angehörten, Verbindung aufzunehmen; sie haben von diesem Recht Gebrauch gemacht, so gilt dies auf keinen Fall für die Vertreter der Geographie, die man immer wieder mit ausweichenden Antworten vertröstete.

Im Herbst 1964 erschien der Vorentwurf für die Abänderung des Maturitätsreglementes. Er enthielt eine Menge Vorschläge verschiedenster Art. Wir beschränken uns auch hier auf diejenigen Punkte die das Fach Geographie betreffen. Wie für Biologie, Chemie und bei den Typen A und B auch für Physik darf nun auch für die Geographie der Fachunterricht nicht früher als zwei Jahre vor dem Ende der gesamten Schulzeit abgeschlossen werden. Mit diesem Vorschlag mußten sich die Geographen, wenn auch schweren Herzens, notged rungen abfinden.

Fast schwerwiegender war ein anderer Punkt, der vieldiskutierte Artikel $13^{4}$, der das Stundenverhältnis zwischen den sprachlich-historischen und den mathematisch-naturwissenschaftlichen Fächern regelt. Er lautet: Bei allen Typen soll zwischen den Fächergruppen der sprachlich-historischen Richtung und der mathematisch-naturwissenschaftlichen Richtung ein ausgewogenes Verhältnis herrschen; daher darf der Anteil am gesamten Aufwand obligatorischer Stunden dieser beiden Fächergruppen für die sprachlich-historischen Fächer (Sprachen, Geschichte, Philosophie, Kunstgeschichte und Staatskunde) nicht unter der Hälfte, für die mathematisch-naturwissenschaftlichen Fächer (Mathematik, Physik, Chemie, Biologie und deren Praktika) nicht unter einem Viertel liegen.> Die Geographie ist als einziges obligatorisches Fach in dieser Fächergruppe nicht genannt. Dafür werden zur sprachlich-historischen Richtung nicht obligatorische Fächer wie Philosophie, Kunstgeschichte und Staatskunde gezählt. Die Zwitterstellung der Geographie ist ihr einmal mehr zum Verhängnis geworden! Mehrere Gremien, unter anderen auch der Gymnasiallehrerverein, hatten sich zur neuen Maturitätsanerkennungsverordnung zu äußern. Letzterer behandelte den ganzen Fragenkomplex in der Form eines erweiterten Vorstandes, dem vor allem je ein zweiter Fachvertreter angehörte, in zwei Sitzungen (ganztägig am 20. Februar und halbtägig am 6. März 1965) in Olten. Zur Besprechung einer Stellungnahme unserer beiden Vertreter (Bächler, St. Gallen, und Bernhard, Zürich) im Gymnasiallehrerverein trafen sich vorgängig folgende Geographen in der Eidgenössischen Technischen Hochschule: H. Bächler, H. Bernhard, H. Boesch, E. Egli, H. Gutersohn, H. Hofer, Ed. Imhof, W. Nigg und E. Winkler. Sie einigten sich schließlich auf folgenden Zusatz zu Artikel $13^{4}$ ¿Die Geographie kann zur einen oder anderen Fächergruppe gerechnet werden; ihre Stundenzahl soll einen $Z_{\text {wanzigstel aller obligatorischen } S \text { tunden nicht unterschreiten.> }}$ Dieser Anteil entspricht ungefähr der bisherigen Zuteilung.

Die erste Vorstandssitzung des Gymnasiallehrervereins, in der Artikel $13^{4}$ diskutiert wurde, verlief gar nicht nach Wunsch der beiden Fachvertreter. Vorerst wurde ein Zusatz folgenden Inhaltes beschlossen: «Die Geographie ist mit Rücksicht auf ihre Sonderstellung in die Berechnung dieses Verhältnisses nicht einbezogen.> Das wäre mit dem vorgesehenen Zusatzpassus über die Stundenzahl ohne weiteres akzeptabel gewesen. Aber dieser wurde von der Versammlung, trotz den wohlfundierten Voten der beiden anwesenden Geographen, mit $48 \mathrm{zu} 3$ (beide Fachvertreter und ein wohlwollender Historiker) Stimmen und bei 8 Enthaltungen wuchtig verworfen. Es war ein Plenum mit lauter Fachinteressen, das wenig Verständnis für die berechtigten Forderungen der Geographen zeigte und bei dem selbst der Präsident Partei gegen die erhobene Forderung der Geographen bezog.

Natürlich bedeutet die Stellungnahme des Gymnasiallehrervereins noch keinen Entscheid. Aber er hätte dem Begehren der Geographen doch starken Nachdruck verliehen.

Es blieb uns nichts anderes übrig, als möglichst viele Mitglieder der Eidgenössischen Maturitätskommission auf diesen Mißstand aufmerksam zu machen, haben doch diese 
Männer ein gewichtiges Wort, beinahe das letzte, mitzusprechen. Hier erwies sich Herr Prof. Gutersohn wieder einmal als unser hilfsbereiter Freund.

Es bleibt nun abzuwarten, was die Eidgenössische Maturitätskommission und das Departement des Innern beschließen. Verzögert wurde die ganze Angelegenheit durch die Ärztesohaft, die sich auf einer Versammlung mit einem Mehr von nur einer Stimme für das Wegfallen der Lateinergänzungsprüfung für Typus $\mathrm{C}$ entschließen konnte und nachträglich eine von Ärztekreisen geforderte Urabstimmung durchführen mußte. Man kann sich mit Recht fragen, worauf diese erstaunliche Machtstellung der Ärzteschaft in der Maturitätsfrage beruht. Ständerat Vaterlaus schreibt in seinem oberwähnten Artikel vom 3. Juli 1964: «Es muß auch als unverständlich bezeichnet werden, daß gerade nur der Stand der Ärzte, Zahnärzte, Tierärzte und Apotheker das Privileg beansprucht und darin durch den Bund geschützt werden soll, daß alle seine Angehörigen des Lateins mächtig sein müssen, während andere, nicht minder wichtige Berufe sicher an die geistige Beweglichkeit und an die Verantwortung den Mitmenschen gegenüber keine geringeren Ansprüche stellen.> Die Erklärung gibt Prof. Schmid in seiner Erläuterung über die formelle Bedeutung der MAV (Maturitäts-Anerkennungsverordnung) und ihre faktische Tragweite: ¿Die Expertenkommision war sich bei ihrer Arbeit der eigentümlichen Tatsache immer bewußt, daß der Bund den Schulen nur im Hinblick auf den genau bestimmten Bereich der Medizinalstudien Vorschriften machen kann, daß aber die zu diesem $Z$ wecke geschaffene MAV eine viel weiter reichende Bedeutung besitzt. Weil jede Mittelschule, die damit rechnet, daß regelmäßig ein Teil ihrer Abiturienten sich für ein medizinisches Studium entscheiden wird, den Anforderungen der MAV genügen will, um diesen Schülern die Ablegung einer besonderen Maturitätsprüfung zu ersparen, kommt es zu der großen faktischen Tragweite dieser Verordnung.) - Auch der Artikel $13^{4}$ geht schließlich auf die Ärzte zurück, heißt es doch in der Erklärung zum Entwurf: «Die Expertenkommission hat gezögert, ob sie eine solche zahlenmäßige Bestimmung des Verhältnisses der Fächergruppen zueinander formulieren wolle ... Wenn die Expertenkommission dennoch diesen Absatz $13^{4}$ so vorlegt und ihm große Bedeutung beimißt, war für sie erstens der dringende Wunsch der Ärztevertreter nach einer konkreten Sicherung des humanistischen Charakters des Typus $\mathrm{C}$ ausschlaggebend.>

Ein weiterer Artikel der vorgesehenen Maturitätsverordnung muß noch kurz erwähnt werden. Er regelt die Notengebung, und zwar so, daß diejenigen der vier Kernfächer (je nach Typus etwas verschieden) den Koeffizienten Zwei erhalten, während alle übrigen Fächer nur einfach bewertet werden. In der obgenannten Vorstandssitzung des Gymnasiallehrervereins wurde dieser Artikel verworfen, nicht zuletzt deshalb, weil man glaubte, sich über die Wahl der mit dem Koeffizienten $Z$ wei bedachten Fächer nicht einigen zu können.

Es ist ganz klar, daß die Stellung der Geographie innerhalb der vorgesehenen neuen Maturitätsverordnung wesentlich geschwächt ist. Natürlich haben die Schulleitungen bei der Ausgestaltung der Lehrprogramme noch ein gewichtiges Wort mitzureden. An den Geographielehrern aber liegt es, bei ihren Schulleitungen und innerhalb der Konvente für ihre gute Sache zu werben und die bisherige Position, die durch die neue Maturitätsverordnung nicht mehr so gestützt wird, zu halten. Zwar bilden die Geographen mit ihrem $Z$ weistundenfach innerhalb der Konvente eine kleine Minderheit, die auf das Wohlwollen ihrer Kollegen angewiesen ist. Hier ergeben sich aber verschiedene Möglichkeiten, gute Freunde und Verständnis für das Fach zu gewinnen. Diese zu pflegen und weiter auszubauen, muß unser großes Anliegen sein.

Wenn wir bedenken, daß in Österreich, Italien, Frankreich, Belgien, Schweden, Finnland, Island, der Türkei, zum Teil auch in England und in Deutschland die Geographie mit ein bis zwei, ja sogar drei (Österreich) Wochenstunden bis zum Schluß der gesamten Schulzeit weitergeführt wird, sehen wir deutlich, wie berechtigt unsere Forderungen, an denen unbedingt festzuhalten ist, sind. 


\title{
QUELLEN
}

Reglement für die Eidg. Maturitätsprüfungen vom 2o. Januar 1925 (Stand Dezember 1954). - Revision der Eidg. Verordnung über die Anerkennung der Maturitätsausweise. Bericht der Eidg. Expertenkommission. - Artikel Vaterlaus in NZZ 28. April 1959 und 3. Juli 1964. H. Bernhard: Der Geographieunterricht in der Schweiz in 'Geographische Rundschaus, März 1963. - H. Bernhard: Die Geographie in den Stundentafeln verschiedener Länder Europas in ‘Geographica Helvetica) 1965.

\section{EINE INTERNATIONALE ARBEITSGRUPPE FÜR DIE GEOGRAPHISCHE TERMINOLOGIE DER AGRARLANDSCHAF'T}

\author{
WERNER GALLUSSER und HAROLD HAEFNER
}

Unter Leitung von Prof. Dr. H. Uhlig, Geographisches Institut der Universität Gießen, hat sich eine internationale Arbeitsgruppe gebildet, der alle agrargeographisch interessierten deutschen Geographen und zahlreiche Vertreter aus 25 weiteren Ländern angehören. Das Ziel dieser internationalen Gemeinschaftsarbeit besteht in der Erstellung eines Glossars, das alle agrargeographischen Fachausdrücke definiert [nach a) der erstmals verwendeten Fassung oder einem Standardwerk und b) dem neuesten Stand der Forschung], alternative Begriffe (regionale Varianten) und den entsprechenden fremdsprachigen Terminus nennt, oder aufzeigt, was unter einem bestimmten Begriff in einem Sprachgebiet verstanden wird.

Wer um die begriffliche Verwirrung weiß und die Gefahr des Aneinander-vorbei-Redens kennt (denken wir nur an den Ausdruck (Transhumanz)), der wird es freudig begrüßen, daß ein derartiges Werk an die Hand genommen wird.

Um eine Vorstellung über Aufbau und Umfang des geplanten Glossars geben zu können, fügen wir die vorgeschlagene provisorische Aufgliederung der Begriffe nach Sachgruppen in einem Dezimalsystem an:

o Allgemeine Grundbegriffe der Kulturforschung, der Agrargeographie und der Agrargeschichte.

o.1 Kulturwissenschaftliche Grundbegriffe (Wirtschafts- und Kulturstufen usw.).

o.2 Agrargeographische Grundbegriffe (Landwirtschaft, Agrargeographie).

o.3 Agrargeschichtliche Grundbegriffe (Villikation, Zehnt).

1 Agrargeographische Landschaftstypen, -zonen, -grenzen usw.

1.o Agrageographisch relevante (natürliche oder anthropogen gewandelte) Landschaftstypen und typische Standorte (Marsch, Puszta).

1.1 Kulturgeographisch-genetische Landschaftstypen (Altsiedelland, Rodungsgebiet).

1.2 Agrargeographische Zonen (kont. Sommerweizenzone, Alpine Stufe).

1.3 Agrargeographisehe Grenzen (Trockengrenze, Höhengrenze der Dauersiedlung).

2 Siedlungen und Siedlungsentwicklung.

2.o Siedlungsformen (Weiler, Angerdorf).

2.1 Funktionelle Siedlungstypen (Sommerdorf, Alpsiedlung, Weingut).

2.2 Siedlungsgenetische Begriffe, Landerschließung und Landgewinnung (Aussiedlerhof, Landnahme, Wüstung).

2.3 Hausformen, -typen, -teile (Vierseithof, Gotthardhaus).

3 Flur- und Nutzflächengefüge.

3.o Gemarkungsorganisation, Besitz- und Nutzungsformen bzw. -areale (Gemarkung, Allmende).

3.1 Flurformen und Flurteile (Zelge, Blockflur).

3.2 Besitzmaße und -einheiten und Ackermaße (Hufe, Tagewerk).

3.3 Intensitäts-, Anbau-, Flurzonen (Wildland, Thünensche Ringe).

3.4 Einhegungen, Feld- und Flurgrenzen (Windschutzstreifen, Etter).

4 Landnutzung.

4.o Landwirtschaftliche Betriebsformen (Gemischtbetriebe, Grünlandwirtschaft).

4.1 Wechselwirtschaftsformen (shifting-cultivation, Egartenwirtschaft).

4.2 Viehwirtschaft (Viehzucht, Transhumanz, Nomadismus).

4.3 Ackerbau (Dreifelderwirtschaft, Regenfeldbau).

4.4 Künstliche Bewässerung (Bewässerungswiesen, Schöpfrad, bisse) 